\title{
Antibiotic prescription practices and their relationship to outcome in South African intensive care units: Findings of the Prevalence of Infection in South African Intensive Care Units (PISA) Study
}

\author{
Fathima Paruk, Guy Richards, Juan Scribante, Sats Bhagwanjee, Mervyn Mer, Helen Perrie
}

Background. The emergence of multidrug-resistant, extensively resistant and pan-resistant pathogens and the widespread inappropriate use of antibiotics is a global catastrophe receiving increasing attention by health care authorities. The antibiotic prescription practices in public and private intensive care units (ICUs) in South Africa are unknown.

Objective. To document antibiotic prescription practices in public and private ICUs in South Africa and to determine their relationship to patient outcomes.

Methods. A national database of public and private ICUs in South Africa was prospectively studied using a proportional probability sampling technique.

Results. Two hundred and forty-eight patients were recruited. Therapeutic antibiotics were initiated in 182 (73.5\%), and 54.9\% received an inappropriate antibiotic initially. De-escalation was practised in $33.3 \%$ and $19.7 \%$ of the public and private sector patients, respectively. Antibiotic duration was inappropriate in most cases. An appropriate choice of antibiotic was associated with an $11 \%$ mortality, while an inappropriate choice was associated with a $27 \%$ mortality $(p=0.01)$. The mortality associated with appropriate or inappropriate duration of antibiotics was $17.6 \%$ and $20.6 \%$, respectively $(p=0.42)$.

Conclusion. Inappropriate antibiotic prescription practices in ICUs in the public and private sectors in South Africa are common and are also associated with poor patient outcomes.

S Afr Med J 2012;102(7):613-616
Antibiotics are commonly prescribed to critically ill patients throughout the world, with rates as high as $60 \%{ }^{1}$ A major concern is the almost universal observation that $31-77 \%$ of these are inappropriate..$^{1-5}$ The inappropriate use of antibiotics is associated with increased morbidity, mortality and cost, and is a major driver in the emergence of resistant pathogens. Antibiotic prescription practices in South African intensive care units (ICUs) have not been described in the public or private sectors. Apart from its economic implications, this information is relevant because of the emergence of extremely high levels of drug resistance among Gram-negative bacilli in South Africa. ${ }^{6}$

As a continuum of the National Critical Care Audit, the Critical Care Society of Southern Africa (CCSSA) undertook a 1-day prevalence study of infection as a first step to ascertain the national profile of sepsis among critically ill patients..$^{711}$ One aspect of the study was to ascertain antibiotic prescription practices in South African ICUs and to determine their relationship to patient outcome.

Department of Anaesthesiology, Faculty of Health Sciences, University of the Witwatersrand, Johannesburg

Fathima Paruk, MB ChB, MD, FCOG (SA), Crit Care (SA)

Juan Scribante, MCur

Helen Perrie, MSc (Nursing)

Department of Critical Care, Faculty of Health Sciences, University of the Witwatersrand, Johannesburg

Guy Richards, MB BCh, PhD, FCP (SA), FRCP, FCCP

Mervyn Mer, MB BCh, Dip PEC (SA), FCP (SA), MMed (Int Med), FCCP, FRCP,

Cert Crit Care (SA)

Department of Anesthesiology, University of Washington, Seattle, USA Sats Bhagwanjee, FCA (SA) (Crit Care)
Permission to conduct the study was obtained from the ethics committees of the universities of Cape Town, the Free State, KwaZuluNatal, Pretoria, Stellenbosch, Transkei and the Witwatersrand and the Medical University of South Africa. Approval was also obtained from the relevant health authorities including the Department of National Health, the South African National Defence Force, respective provincial health departments and private hospital groups. Approval was additionally obtained from management and CEOs of the respective hospitals.

\section{Methods}

This was a three-part prospective, descriptive study that included a 1-day point-prevalence study to provide a 'snapshot' of events in the ICU. ${ }^{12}$ The primary purpose was to gain maximal information about the characteristics within each specified field. While there was no manipulation of variables, some control over extraneous variables was applied. ${ }^{13}$ The study population comprised public and private sector hospitals in South Africa that were included in part I of the National Critical Care Audit. ${ }^{7}$ To ensure a true South African representation, all adult and paediatric ICUs in the private and public (tertiary, regional and community level) sectors were included.

Patients provided signed, informed consent for their data to be used. Where the patient was unable to give consent, this was referred to their legal representative, or as a last resort consent was obtained from an attending clinician not involved with the study. Patient and unit confidentiality was maintained.

Proportional probability sampling was used for randomisation. Based on results from the national audit, ${ }^{9}$ an estimated infection prevalence of $15 \%$ and estimated bed occupancy of $90 \%$, a $10 \%$ sample was deemed representative of the study population and acceptable within the study's financial and human constraints. It was determined that a 240-patient sample was attainable from 43 ICUs (mean 6 beds per unit), and from this sample every 8th bed was included and taken to be representative of that particular ICU. Given 
the differences between private and public ICUs, the results were separated into two cohorts.

The managers of the hospitals involved were asked to identify a fieldworker capable of collecting data from their ICU. The researchers assisted those ICUs that could not identify a fieldworker. A draft 24-page data collection sheet, based on the literature and the researchers' expertise, was presented to the CCSSA council for discussion, and feedback was incorporated into the final data sheet.

The fieldworkers, all medical doctors or registered nurses working in ICUs, underwent a 4-hour training workshop before the study. Each fieldworker received a small monetary sum per patient recruited.

Study documents were couriered or hand-delivered to the units shortly before the designated study day. On 16 August 2005 all patients who were in the selected units between $00 \mathrm{~h} 00$ and $24 \mathrm{~h} 00$ were eligible for recruitment. The following day fieldworkers collected part I (unit demographics) and part II (pre-study day and study day) data. Organ function scores were noted for a further 5 days or until the patients were discharged from the ICU, whichever came first. Documentation was returned to the researchers by courier. Fieldworkers subsequently collected part III (ICU and hospital discharge dates and whether or not the patients survived to discharge) up to a period of 30 days after recruitment. This was completed on 16 September 2005, and the completed data sheets were returned by facsimile.

The signed consent forms and patient identification lists were checked for completeness by two unblinded researchers. The respective documentation from each unit was then sealed and filed separately. The remaining study documentation was then reviewed by one of two researchers. If discrepancies were found, the appropriate contact person was requested to confirm or correct the data or, as occurred with four units, two researchers provided on-site assistance to fieldworkers who had difficulty obtaining all the data. Subsequently two researchers entered the data into a pre-prepared Microsoft Excel data sheet with multiple data integrity checks. Copies of microbiology results (with redacted patient identifiers) were submitted together with each patient's study documentation. The microbiology results were reviewed and interpreted by an expert microbiologist.

Three certified intensivists from Charlotte Maxeke Johannesburg Academic Hospital analysed the adult data with respect to the diagnosis of sepsis. Antibiotic prescription practices were assessed by two intensivists. Where there was a lack of agreement, a third intensivist was involved and the issue discussed until consensus was obtained.

The data captured included the number of patients prescribed therapeutic antibiotics, whether the choice was appropriate, whether de-escalation and duration of administration were appropriate, and the hospital mortality. The choice was regarded as appropriate if the suspected micro-organism was usually sensitive to the empiric choice and the antibiotic was prescribed according to an acceptable regimen (loading dose, dosage and dosing interval). De-escalation was considered appropriate if it was achieved within 72 hours of empiric antibiotic initiation or within 24 hours of identification of the micro-organism and the susceptibility result. Inappropriate duration of therapy was defined as an unacceptably prolonged duration. Given the lack of evidence on this issue, we were flexible, only considering it to be inappropriate if without good reason it exceeded 10 days (lower respiratory tract infections), 14 days (abdominal sepsis), 7 days (urinary tract infections), 6 weeks (osteomyelitis, infective endocarditis), or 3 weeks post negative culture (antifungal therapy).

If a patient received antibiotics on more than one occasion during the study period, the choice of antibiotic had to be appropriate on all three occasions to be captured as an appropriate choice. The same principle was applied to determine appropriate duration.

\section{Results}

Of the 248 patients recruited (Table I), $65.7 \%$ were from the private sector; $69(27.8 \%)$ had evidence of sepsis, severe sepsis or septic shock on the study day, and 196 were deemed to have sepsis by the primary physician, representing an over-diagnosis of 51\%. From the study day until day 30 or discharge, empiric antibiotics were initiated in 182 (73.5\%) patients; 100 (54.9\%) received inappropriate antibiotics. The majority were initiated for respiratory $(65.2 \%)$, abdominal (16.7\%), urogenital (6.9\%) and skin, soft-tissue and wound infections (6.9\%). An inappropriate antibiotic was prescribed in 27 (43.5\%) and 73 $(60.8 \%)$ of the public and private sector patients, respectively. All patients who were prescribed antibiotics received an average of 3 agents during their stay. De-escalation was practised in $33.3 \%$ and $19.7 \%$ of the public and private sector patients, respectively. Antibiotic duration was inappropriate in $53.2 \%$ and $81.7 \%$ of the public and private sector patients, respectively.

The number of anti-infective agents prescribed simultaneously to each patient ranged from 1 to 10 (Fig. 1). This excluded antituberculosis or antiviral agents but included antifungal agents. As an example, one patient simultaneously received cloxacillin, teicoplanin, metronidazole, amikacin, ceftazadime, meropenem, levofloxacin, fluconazole, erythromycin and sulfamethoxazole/trimethoprim. The primary reason for the large number of simultaneous antibiotics was a tendency not to stop previous therapies on initiation of a new antibiotic.

Of the patients who received antibiotics, $36(19.8 \%)(n=182)$ died. An appropriate antibiotic choice was associated with an $11 \%$

Table 1. Antibiotic prescription in South African intensive care units

\begin{tabular}{llll}
\hline & $\begin{array}{l}\text { Public } \\
(\boldsymbol{n}(\%))\end{array}$ & $\begin{array}{l}\text { Private } \\
(\boldsymbol{n}(\%))\end{array}$ & $\begin{array}{l}\text { All } \\
(\boldsymbol{n}(\%))\end{array}$ \\
\hline Patients & $85(34.3)$ & $163(65.7)$ & 248 \\
$\begin{array}{l}\text { Antibiotics } \\
\text { prescribed }\end{array}$ & $62(72.9)$ & $120(73.6)$ & $182(73.4)$ \\
$\begin{array}{l}\text { Inappropriate } \\
\text { empiric } \\
\text { antibiotic }\end{array}$ & $27(43.5)$ & $73(60.8)$ & $100(54.9)$ \\
$\begin{array}{l}\text { De-escalation } \\
\text { practised }\end{array}$ & $9 / 27(33.3)$ & $12 / 61(19.7)$ & $21 / 88(23.9)$ \\
$\begin{array}{l}\text { Inappropriate } \\
\text { duration of } \\
\text { antibiotics }\end{array}$ & $33(53.2)$ & $98(81.6)$ & $131(72.0)$ \\
& & &
\end{tabular}

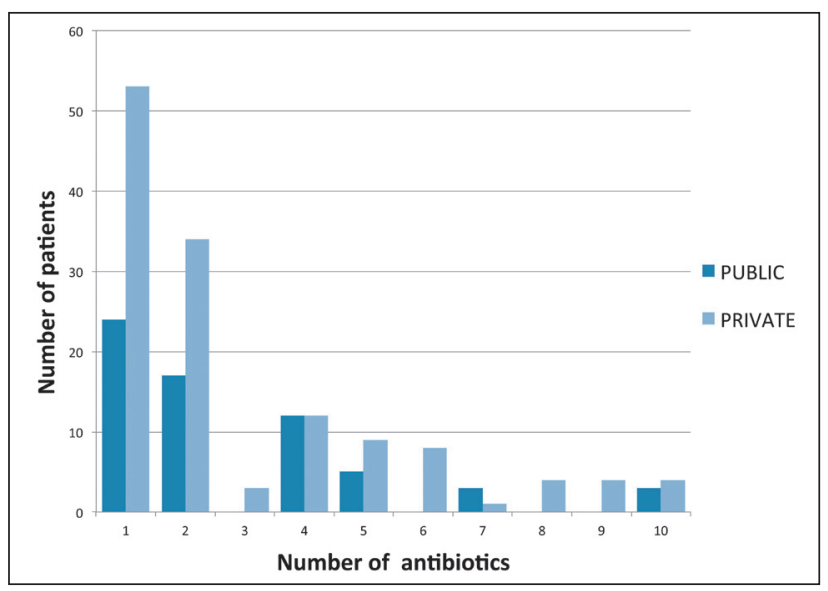

Fig. 1. Simultaneous prescription of antibiotics. 
mortality, while an inappropriate choice was associated with a $27 \%$ mortality ( $p=0.01$ ) (Fig. 2). The mortality associated with appropriate or inappropriate duration of antibiotics was $17.6 \%$ and $20.6 \%$, respectively $(p=0.42)$.

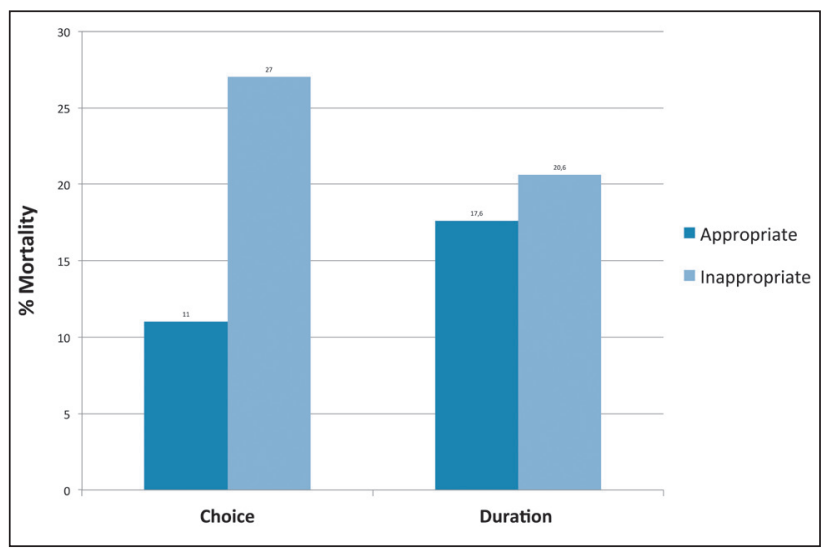

Fig. 2. Mortality associated with appropriate and inappropriate choice and duration of antibiotics.

\section{Discussion}

The antibiotic prescription rate of $73.4 \%$ is high, but comparable to that observed globally. ${ }^{1,14,15}$ The larger representation of the private sector is in keeping with the availability of ICU beds relative to the public sector in South Africa.

The initiation of an appropriate empiric antibiotic has been demonstrated to confer a mortality benefit in various studies and constitutes a crucial element in the treatment of infections. ${ }^{2,4}$ Kumar et al. recently reported that inappropriate antibiotic prescription results in a fivefold mortality increase in septic shock. ${ }^{16}$ It is cause for concern that an inappropriate antibiotic is initiated in approximately $55 \%$ of patients. While inappropriate prescription rates ranging from $31 \%$ to $77 \%$ have previously been reported, this is nevertheless unacceptable in an environment of increasing drug resistance..$^{1-5}$ The higher rate in the private sector might be explained by the greater number of 'open' ICUs in this sector where the lack of a director permits all attending doctors to prescribe antibiotics as and when they wish, often without sufficient knowledge of the pharmacology of the drugs or the epidemiology of the unit.

Duration of antibiotic use was inappropriate in $72 \%$ of patients in this study. Although not associated with increased mortality this is extremely worrying, as this practice is associated with increased development of resistance to antimicrobials. ${ }^{17} \mathrm{Of}$ particular concern is the emergence of highly resistant Gramnegative pathogens, where the spectrum of therapeutic options is rapidly shrinking. De-escalation was similarly seldom practised, which also impacts on cost and resistance. Many patients receiving multiple (more than 4) antibiotics simultaneously is also unacceptable. These findings demonstrate the lack of knowledge and insight regarding antibiotic use prevalent among doctors who work in ICUs and as such are probably among the best informed regarding antibiotic use in the South African medical community.

Antibiotic prescription practices in South African ICUs are far from acceptable. Consequently it has become necessary to revert to the use of older, more toxic agents such as colistin and fosfomycin, as no new antibiotics have become available, or will become available in the foreseeable future. It is crucial that antibiotic stewardship becomes mandatory in South Africa's ICUs and that this should extend to the prescribing community as a whole. In particular, limitations must be placed on the use of broadspectrum agents if we are still to be able to treat infections in $5-10$ years' time. Poor prescribing practices are also associated with poor fiscal outcomes, increased mortality and further limitation of therapeutic options.

\section{Limitations}

Fieldworkers had varying research experience, but most were research novices. We attempted to address this by using only fieldworkers with ICU experience, and by providing a mandatory pre-study training workshop and an extensive written study guideline. In addition, the investigators were available for consultation at all times. We acknowledge that the classification of whether an antibiotic is appropriate or not is difficult, as has been the case in other studies. Re-analysis of data by the same group of experts also does not always reproduce the same observations. However, the investigators opted to utilise accredited intensivists from a tertiary academic institution as it was felt that they were sufficiently well versed with public and private sector ICU antibiotic prescription practices, the microbiological profiles of organisms cultured and antimicrobial resistance patterns nationally. We acknowledge that this may not be ideal, but believe that it was a better strategy than utilising multiple intensivists from all the provinces.

\section{Conclusion}

This study was a crucial step toward understanding antibiotic prescription practices in South African ICUs. The study is particularly relevant because of the current increase in resistance and it gives impetus to a national drive toward antibiotic stewardship. To achieve these aims it was vital that a representative sample with reliable data was obtained. We believe that the study design ensured rigour and good quality control with adequate data validation processes.

\section{Key recommendations}

There is a need for a national educational programme regarding the use of antibiotics in critically ill patients. This should include instruction to prescribers on the following:

- The role of inappropriate antibiotic prescription practices in the development of multidrug resistance

- The need to ascertain and be aware of the most frequent organisms causing hospital- and community-acquired sepsis and their susceptibilities

- The diagnosis of sepsis, including the necessity for frequent cultures

- The rational use of antibiotics, including empiric choice, dosing strategies, de-escalation and duration of treatment.

While the above has the potential to improve current practice, there is no evidence that education alone translates into improved practice. Drastic measures are therefore required to curtail the irrational use of antibiotics. In order to preserve our existing resources, we believe that it is ethically justifiable to consider restricted use of antibiotics and punitive measures for those failing to comply.

Disclosures. This project was funded by an unrestricted research grant from the CCSSA.

Acknowledgements. The authors express their gratitude to the CCSSA council, Professor Piet Becker, the CEOs of all the participating hospitals, the directors of the private hospital groups, and the fieldworkers who collected data. 


\section{References}

1. Erbay A, Bodur H, Akinci E, Colpan A. Evaluation of antibiotic use in intensive care units of a tertiary care hospital in Turkey. J Hosp Infect 2005;59(1):53-61. [http://dx.doi:10.1016/j.jhin.2004.07.026] 2. Raineri E, Pan A, Mondello P, et al. Role of the infectious diseases specialist consultant on the appropriateness of antimicrobial therapy prescription in an intensive care unit. Am J Infect Control 2008;36:283-290. [http://dx.doi.org/10.1016/j.ajic.2007.06.009]

3. Von Gunten V, Reymond JP, Boubaker K, et al. Antibiotic use: is appropriateness expensive? J Hosp Infect 2009;71(2):108-111. [http://dx.doi.org/10.1016/j.jhin.2008.10.026]

4. Luna CM, Aruj P, Niederman MS, et al. Appropriateness and delay to initiate therapy in ventilatorassociated pneumonia. Eur Respir J 2006;27(1):158-164. [http://dx.doi.org/10.1183/09031936.06.000 49105.

5. Shorr AF, Micek ST, Kollef MH. Inappropriate therapy for methicillin-resistant Staphylococcus aureus: resource utilization and cost implications. Crit Care Med 2008:36(8):2335-2340. [http://dx.doi. org/10.1097/CCM.0b013e31818103ea]

6. Brink A, Feldman C, Richards GA, et al. Emergence of extensive drug resistance (XDR) among Gramnegative bacilli in South Africa looms nearer. S Afr Med J 2008;98:586-592.

. Scribante J, Bhagwanjee S. National audit of critical care resources in South Africa - research methodology. S Afr Med J 2007;97(12 Pt 3):1308-1310. Epub 13 February 2008.

8. Bhagwanjee S, Scribante J. National audit of critical care resources in South Africa - unit and bed distribution. S Afr Med J 2007;97(12 Pt 3):1311-1314. Epub 13 February 2008.

9. Scribante J, Bhagwanjee S. National audit of critical care resources in South Africa - open versus closed intensive and high care units. S Afr Med J 2007;97(12 Pt 3):1319-22. Epub 13 February 2008.

10. Scribante J, Bhagwanjee S. National audit of critical care resources in South Africa -nursing profile. S Afr Med J 2007;97(12 Pt 3):1315-1318. Epub 13 February 2008
11. Scribante J, Bhagwanjee S. National audit of critical care resources in South Africa - transfer of critically ill patients. S Afr Med J 2007;97(12 Pt 3):1323-1326. Epub 13 February 2008.

12. Katzenellenbogen IM Joubert G Abdool Karim SS. Epidemiology: A Manul for South Africa. 1st ed. Cape Town: Oxford University Press, 2002.

13. Burns N, Grove SK. The Practice of Nursing Research: Appraisal, Synthesis and Generation of Evidence. 6th ed. Philadelphia: Saunders, 2009

14. Vincent JL, Rello J, Marshall J, et al. International study of the prevalence and outcomes of infection in intensive care units. JAMA 2009; 302(21):2323-2329. [http://dx.doi.org/10.1001/jama.2009.1754] 5. Porretta A, Giuliani L, Vegni FE, et al. Prevalence and patterns of antibiotic prescribing in Italian hospitals. Infection 2003;31(Suppl 2):16-21.

16. Kumar A, Ellis P, Arabi Y, Roberts D, et al. Initiation of inappropriate antimicrobial therapy results in a fivefold reduction of survival in human septic shock. Chest 2009;136(5):1237-1248. [http://dx.doi. org/10.1378/chest.09-0087]

17. Chastre J, Wolff M, Fagon JY, et al. Comparison of 8 vs 15 days of antibiotic therapy for ventilatorassociated pneumonia in adults: a randomized trial. JAMA 2003;19:290(19):2588-2598. [http://dx.doi. org/10.1001/jama.290.19.2588]

Accepted 13 June 2012. 